\title{
New variations on the theme
}

\section{J. Farr and P. N. Goodfellow}

THE latest results from Alec Jeffreys' laboratory (page 204 of this issue ${ }^{1}$ ) will attract the close attention of patent officers and the legal profession, as well as scientists. The paper reports a new DNA-fingerprinting technique which extends existing methods by assaying sequence variation within minisatellite arrays.

Minisatellites are hypervariable regions of the genome which exhibit polymorphism due to a variable number of tandem repeats of a short sequence. In 1985 , Jeffreys and co-workers ${ }^{2-4}$ demonstrated that a DNA 'polycore' probe based on a myoglobin gene minisatellite can simultaneously detect, by hybridization to human genomic DNA, a large number of dispersed hypervariable loci containing tandem repeats of similar sequence. The complex restrictionfragment pattern that results can produce a DNA fingerprint specific to an individual (the only exception being monozygous twins), and the applications have been legion.

To improve the sensitivity of multilocus DNA fingerprint analyses, the next step was amplification of human minisatellites by the polymerase chain reaction (PCR). Perhaps surprisingly, in view of their highly repetitive nature, it was found that Taq polymerase could amplify entire minisatellites whilst preserving the allelic specificity of the number of repeat units ${ }^{5}$. Moreover, several minisatellites could be co-amplified to generate reproducible PCR-derived DNA fingerprints, increasing sensitivity by several orders of magnitude, and high-fidelity amplification of hypervariable loci in one or a few cells became attainable.

\section{MVR mapping}

Last year PCR was used to analyse allelic variation within a minisatellite locus ${ }^{6}$, until then an unexplored source of human DNA polymorphism. This approach, called minisatellite variant repeat (MVR) mapping, dramatically increases the number of different alleles of any minisatellite locus that can be distinguished in a population. This method has been applied to the hypervariable locus D1S8 (probe MS32) at region 4243 on the long arm of chromosome 1. Previous DNA sequence analyses of cloned MS32 had shown that about half the repeat units share an $A \rightarrow G$ transition which creates an HaeIII restriction site readily amenable to MVR mapping. Interspersion patterns of $\mathrm{HaeNI}^{+}$and $\mathrm{HaeIII}^{-}$repeat units (designated a-type and t-type) were assayed by PCR ampli- fication of an entire allele followed by end-labelling and partial digestion with either HaeIII or HinfI, agarose gel electrophoresis and autoradiography.

The partial digest pattern generated by HinfI, which cuts once within each repeat unit, produces a continuous ladder of labelled fragments from which the number of minisatellites can be determined. Comparison of HaeIII and HinfI partial digests enables each repeat unit to be scored as to whether or not it is cleaved by HaeIII. High levels of allelic variation were revealed, particularly near the beginning of the D1S8 array. MVR mapping provides an unambiguous binary code for any allele, but is technically demanding (for mere mortals at least), limited to alleles small enough to amplify by PCR, and cannot be used for diploid mapping.

Jeffreys et al. ${ }^{1}$ have now come up with a much simpler system, MVR-PCR. It involves the use of two MVR-specific oligonucleotides which differ at their $3^{\prime}$ ends, allowing allele-specific priming of either a-type or t-type repeat units. PCR using one or other of these primers, together with a primer from a fixed site in the minisatellite-flanking DNA, generates two complementary sets of products from which the MVR map can be deduced. To prevent the oligonucleotides priming internally within PCR products, MVR detection and subsequent amplification are uncoupled by providing each MVR-specific primer with a 20-nucleotide 'tag' at the 5' end. Primers complementary to the tag are used for amplification in subsequent cycles.

MVR-PCR can be undertaken on total genomic DNA, where a superimposed profile of both alleles produces a diploid code, and the DNA profiles are generated as extraordinarily variable digital sequences resembling bar codes suitable for computer analysis. The data are highly objective, each experiment being internally controlled. In addition the method is reliable and sensitive, and can be applied to degraded DNA, making it particularly well suited to forensic work. Many of the problems associated with conventional DNA fingerprinting, such as the need for side-by-side comparison of DNA samples of the same gel, are obviated.

But what of the limitations? One is that low-cycle PCR prevents direct visualization of the PCR products in ethidium bromide-stained gels. More serious is that only a few minisatellites have so far been identified that are suitable for analysis by this approach - that is, minisatellites that show internal variation, but do not display variant length repeats (abnormal length repeats would throw the MVR diploid coding ladders out of register and make them uninterpretable). Moreover, the extremely high de novo mutation rate at D1S8 limits the effectiveness of the method in, for example, paternity testing and evolutionary studies, two areas for which, at first glance, it would appear to be ideal. Also, in establishing family relationships, the possibility of error arises from the need to identify heterozygous 'null' positions from interpretation of band intensities (dosage is important where one allele contains a sequence variant which fails to amplify with either primer).

\section{Allelic variability}

The resolving power of MVR-PCR reveals that there can be very high levels of allelic variability at human minisatellites and provides the first evidence that recombination is involved in the generation of ultravariability. Several lines of evidence ${ }^{2,7-9}$ have suggested that minisatellites may be recombination hotspots involved in chromosome pairing or the initiation of meiotic recombination, or both. But the failure to detect unequal crossing-over between homologous chromosomes $^{6,10,11}$ seemed to contradict that notion. The finding ${ }^{1}$ of two apparent recombinants at D1S8 in only 572 meioses tested will re-open debate about the involvement of minisatellites in meiosis and recombination.

In British legal history, the name of Jeffreys has been indelibly associated with the judge at the Bloody Assizes of 1685 , and with arbitrary and brutal 'justice'. His latter-day (presumably unrelated) namesake is bringing scientific objectivity to the law courts. In part as eponymous tribute, in part as a gesture against the rising tide of acronyms, we propose that MVR-PCR should be known as the Jeffreys method.

C. J. Farr and P. N. Goodfellow are in the ICRF Laboratories, Lincoln's Inn Fields, London WC2A 3PX, UK.

1. Jeffreys, A. J., MacLeod, A., Tamaki, K., Neil, D. L. \& Monckton, D. G. Nature, 354, 204-209 (1991)

2. Jeffreys, A. J., Wilson, V. \& Thein, S. L. Nature 314 67-73 (1985)

3. Jeffreys, A. J., Wilson, V. \& Thein, S.L. Nature 316 76-79 (1985)

4. Jeffreys, A. J. Brookfield, J. F. Y. \& Semeonoff, R. Nature 317, 818-819 (1985).

5. Jeffreys, A. J., Wilson, V., Neumann, R. \& Keyte, J. Nucleic Acids Res. 16, 10953-10971 (1988).

6. Jeffreys, A. J., Neumann, R. \& Wilson, V. Cell 60 473-485 (1990).

7. Steinmetz, M., Stephan, D. \& Lindahl, K. F. Cell 44 895-904 (1986)

8. Royle, N., Clarkson, R. E., Wong, Z. \& Jeffreys, A. I Genomics 3, 352-360 (1988).

9. Chandiey, A. C. \& Mitchell, A. R. Cytogenet. Cell. Genet. 48, 152-155 (1988).

10. Wolff, R. K., Nakamura, Y. \& White, R. Genomics 3 347-351 (1988).

11. Wolff, R. K., Plaetke, R., Jeffreys, A. J. \& White, R. Genomics 5, 382-384 (1989). 